Article

\title{
Postponing the Onset and Alleviating the Load of Transonic Buffet by Using Steady and Periodic Tangential Slot Blowing
}

\author{
Huixue Dang ${ }^{1,2, * \mathbb{D}}$, Junhai Zhao ${ }^{1}$, Zhichun Yang ${ }^{2}$ and Huibo Dang ${ }^{3}$ \\ 1 School of Civil Engineering, Chang'an University, Xi'an 710061, China; zhaojh@chd.edu.cn \\ 2 School of Aeronautics, Northwestern Polytechnical University, Xi'an 710072, China; yangzc@nwpu.edu.cn \\ 3 Shaanxi College of Communication Technology, Xi'an 710018, China; zhoulinshijie@126.com \\ * Correspondence: perpetual@mail.nwpu.edu.cn
}

Received: 19 June 2019; Accepted: 16 September 2019; Published: 2 October 2019

check for updates

\section{Featured Application: Transonic buffet alleviation for airplanes.}

\begin{abstract}
Transonic buffet not only influences the structural integrity, handling quality and ride comfort, but also limits the flight envelope of transporters and airliners. To delay buffet onset and alleviate the buffet load, the effects of both steady and periodic tangential slot blowing are investigated. The results show that steady tangential blowing on the airfoil upper surface can postpone the buffet onset margin and evidently increase the lift coefficient at incidence angles near and above the buffet onset case of the clean airfoil. Under buffeting conditions of the clean airfoil, unsteady aerodynamic loads can be greatly suppressed by both steady and periodic blowing. The control effort is depicted as reduced wedge effect and weakened dynamic effect. The buffet mechanism includes (a) the feedback loop between the Kutta wave and the separation bubble under the shock foot, and (b) the interaction between the shear layer shed by the shockwave and Kutta waves. Under blowing conditions, the upstream creeping Kutta waves are prevented, and the intensity of the shear layer shed by the shockwave into separated flows is evidently reduced. Parametric studies show that the control effect is reduced as the blowing slot moves downstream, and steady blowing at $41 \% \mathrm{x} / \mathrm{c}$ is the most favorable control case.
\end{abstract}

Keywords: transonic buffet; tangential slot; steady and periodic blowing; postpone of buffet onset; buffet load alleviation

\section{Introduction}

To save time and energy, high-speed flights are usually done by modern, large commercial aircraft, which always cruise between Mach 0.7 and 0.9 by employing supercritical wings [1-3]. These Mach numbers fall in the typical transonic flow range; consequently, unsteady shockwave/boundary layer interactions could induce intensive, large-scale, unsteady lift, and transonic buffet will be the result [4-10]. This transonic buffet not only decreases riding comfort [10-12], but structural fatigue could also be triggered, and disastrous accidents could even result $[2,4,9,13-15]$. Thus, transonic buffet is deemed one of the most important factors limiting the flight envelope $[2,7,9,14,16,17]$, since a margin of $30 \%$ on the lift coefficient at cruising conditions must be respected by design standards [7].

To delay the buffet onset margin, alleviate buffet loads, and widen the flight envelope, a lot of effort has been devoted to the development, including unveiling buffet mechanisms [4,8,18-22], of functional control techniques $[2,7,9,10,14,16,23,24]$, and so on. Meanwhile, studies have also been conducted to improve measuring techniques employed in wind tunnel tests [25], buffet 
onset prediction methods $[17,26,27]$, numerical simulation techniques of shock/boundary layer interactions $[6,11,25,28-31]$, and so on.

One of the most interesting and difficult research targets is to uncover the mechanism of transonic buffet and shock/boundary layer interactions. Among these studies, wind-tunnel tests as well as numerical simulations, stability analyses, and so on, have been conducted, and different academic viewpoints have been proposed. Although the buffet mechanism has remained inexplicable over six decades due to its complexity [2], research is in progress. For example, in the last five years, Szubert et al. [3] presented that frequency modulation was formed by large-scale, periodic oscillations together with secondary oscillations, where low-frequency, large-scale, periodic oscillations and higher frequency secondary oscillations are generated by buffet instability and the von Kármán mode near the trailing edge of the airfoil, respectively. Timme and Thormann [4] stated that lower-frequency results are similar to the forced-response calculations reported on two-dimensional airfoils, but the three-dimensional shock buffet at higher frequencies is linked to the presence of a global destabilizing mode. Sartor et al. [8] presented that the flow is most receptive to harmonic forcing on the upper surface of the profile; besides the low-frequency shock unsteadiness, the flow also exhibits medium-frequency unsteadiness linked to Kelvin-Helmholtz-type instability. Iovnovich and Raveh [32] pointed out that the pre-buffet aerodynamic resonance response and the change in aerodynamic characteristics are governed by the shock-induced separation phenomenon, as a separation bubble was found behind the shock in static analyses.

In the meantime, investigations on control techniques for mitigating buffet loads have also been performed. This research can be roughly divided into passive control techniques and active control techniques. Passive control techniques include suction slots on upper wing surfaces, vortex generators with inclined angles, three-dimensional bumps, and so on, while research on active controls includes trailing edge deflectors, flapping rudders, and so on.

In the published literature on passive control studies, Thiede and Stanewsky [33] examined the impact of slots ejecting airflow normal to the upper surface of a supercritical airfoil. They figured out that the typical normal shock is modified to be two shocks that form a " $\lambda$ ", which notably decreases the total pressure loss and delays the boundary layer separation induced by the shockwave. Babinsky et al. [34,35] tested the effect of streamwise slots located downstream from the airfoil on the shock/boundary layer interference. They pointed out that by introducing streamwise vortices, streamwise slot control may help delay or prevent downstream separation. By solving Navier-Stokes equations enclosed by an IDDES turbulence model, Huang et al. [1] investigated the influence of vortex generators with inclined angles on the transonic buffet of an OAT15A airfoil. They found that the time-averaged location of the shockwave was pushed downstream; thus, the root-mean-square of the oscillating force in the buffeting zone decreases, and the total lift is enhanced. Molton et al. [36] and Dandois et al. [7] tested mechanical, continuous, and pulsed fluidic vortex generators located upstream from the shock foot to reduce the extent of the separated area, and fluidic actuators as well as mechanical vortex generators have proven to be very efficient in postponing buffet onset. Ogawa et al. [37] proposed a method of using three-dimensional bumps on upper wing surfaces to alleviate the strength of flow interference between the shockwave and boundary layers, and the results indicated that a $30 \%$ decrease of total drag can be achieved for the best control case. Then, Eastwood and Jarrett [38] optimized the three-dimensional bump shape, and they pointed out that the bump can generate stream-wise vortices at high Mach numbers, suggesting improvement in the buffet margin. Tian et al. $[10,24]$ studied the suppression effect of bumps on the transonic buffet. They found that the strength of the shockwave was weakened, and the location of the shockwave was stabilized; thus, buffet and drag-rise characteristics are improved. Liu and Yang [39] suppressed transonic shock oscillation and alleviated the buffet load for supercritical airfoils by using a micro-tab.

Among the active control works on the transonic buffet, Caruana et al. [23,40,41] developed a trailing edge deflector (TED) with a close-circuit control loop to control the transonic buffet. Results showed that when the oscillating frequency of TED was close to buffeting frequency, evident 
control effectiveness could be obtained. The drawback of this method is that the control law for three-dimensional flow is very complicated. Iovnovich and Raveh [32] investigated the control effect of a $20 \%$ chord-length trailing edge flap under prescribed oscillation on transonic buffet, and they found that large, prescribed flap motions eliminated the lift resonance response and significantly reduced the lift coefficient amplitude. Gao et al. [9] tested the impact of flapping rudders on the transonic buffet, and they stated that resonant rudders may be a feasible open-loop strategy to suppress buffet loads under different buffet states. Tian et al. [14] tested the control effect of an upper trailing-edge flap (UTEF), and they pointed out that the UTEF prevents flow separation downstream of the shockwave, shifts the buffet boundary to higher angles of attack, and increases the lift coefficients. Ferman et al. [42] and Abramova et al. [43-46] used tangential slot blowing for transonic buffet control of a P-184-158R supercritical airfoil, and they pointed out that tangential blowing can shift the shockwave downstream and increase the lift.

From the published literature, it is found that by adopting passive control techniques such as slots normal to the wall, bumps and vortex generators on the upper surface, and so on, satisfactory control effects can be obtained under design conditions, while off-design conditions engender unexpected effects, and aerodynamic characteristics can even deteriorate. With the utilization of active control techniques, such as trailing edge deflectors, flapping rudders, and so on, the control law proves to be very complicated for three-dimensional flow, and the size of the control surface is relatively large; thus, large driving forces are needed, which depletes extra energy. In published papers, the blowing intensity has been investigated, and the tangential slot-blowing location and blowing directions, which may influence the control effort, should also be studied. Thus, a tangential slot-blowing control method is investigated to test both steady and periodic blowing control effects based on numerical simulations.

\section{Numerical Methods and Validation}

In the transonic buffet simulation, detached eddy simulation (DES) [1,47-49] and large eddy simulation (LES) [12] series turbulence models can predict three-dimensional buffet flow fields. However, it is not practical to compute various upstream conditions by using DES or LES turbulence models to determine buffet onset boundaries because these methods require a grid resolution that is much higher than that for URANS simulations, resulting in excessively longer computational times. Meanwhile, Soda [50] conceived in their 2007 book that numerical simulations of this phenomenon based on URANS current codes are quite successful in predicting the magnitude and frequency of periodic shock motions during buffet. Thus, in two-dimensional studies of transonic buffet mechanisms and transonic buffet flow controls, URANS $[8,9,13,16,24,30,32,43,44]$ turbulence models such as SA, SST, and so on are widely employed, and favorable results have been obtained. To include transition effects, the transition SA turbulence model [51] was employed in the present study.

The discretization schemes usually employed in compressible Navier-Stokes equations enclosed by URANS turbulence models are HLLC [13,32], Roe [14,16], AUSM + P [8], and AUSP + up [9]. Sartor et al. [8] stated that Roe and Jameson schemes were not considered in their work due to the schemes' poor shock treatment when investigating the stability of the flow, and AUSM+ series schemes were their choice. By improving the AUSM+ scheme for shock-stable and accurate hypersonic heating computations, Kitamura and Shima [52] developed an AUSM + up2 scheme for a wide spectrum of Mach numbers, including hypersonic heating, low speed flow, and 3D aerodynamic applications. This scheme is included in the open source code SU2 [53], and it was employed in the present study to solve the compressible Navier-Stokes equations.

The airfoil profiles widely used in transonic buffet studies are NACA0012 $[9,13,17,21,32,54]$ and OAT15A [1,30,55]. By referring to the well-known NACA0012 2-D airfoil shock-buffet test case by McDevitt and Okuno [54], the NACA0012 airfoil was used in the present study. The steady-state numerical methods were validated by the wind tunnel test results from reference [54]. The free stream Mach number was 0.751 , the Reynolds number was $9.5 \times 10^{6}$, and the incidence angle of the NACA0012 airfoil was $1.99^{\circ}$. The chord length of the NACA0012 airfoil was set to be $1.0 \mathrm{~m}$ in present study. The 
computational domain size and the structured grid is shown in Figure 1. The mesh node numbers on both the upper and lower surfaces of the airfoil were set to be 281, respectively. The far-field boundary was placed at least 50 times that of the chord length. The first grid layer thickness normalized by the chord length was set to be $1 \times 10^{-6}$, corresponding to $y^{+} \approx 1$. The calculated surface pressure agreed well with test results, as plotted in Figure 2.

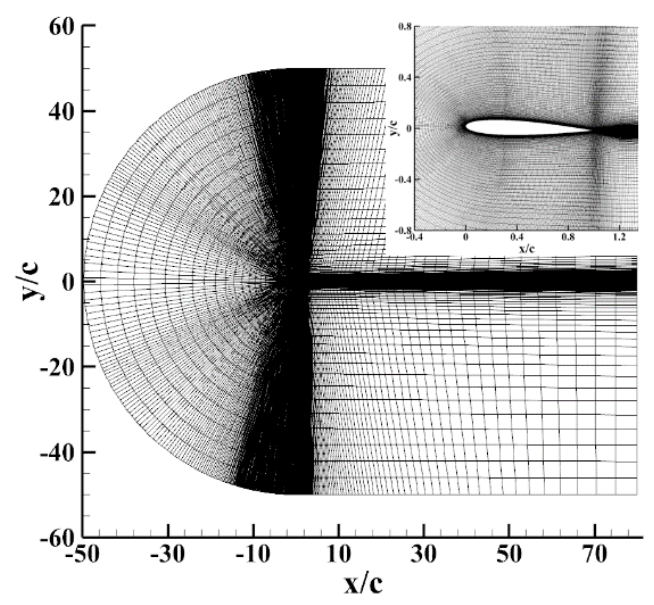

Figure 1. Grid around an airfoil.

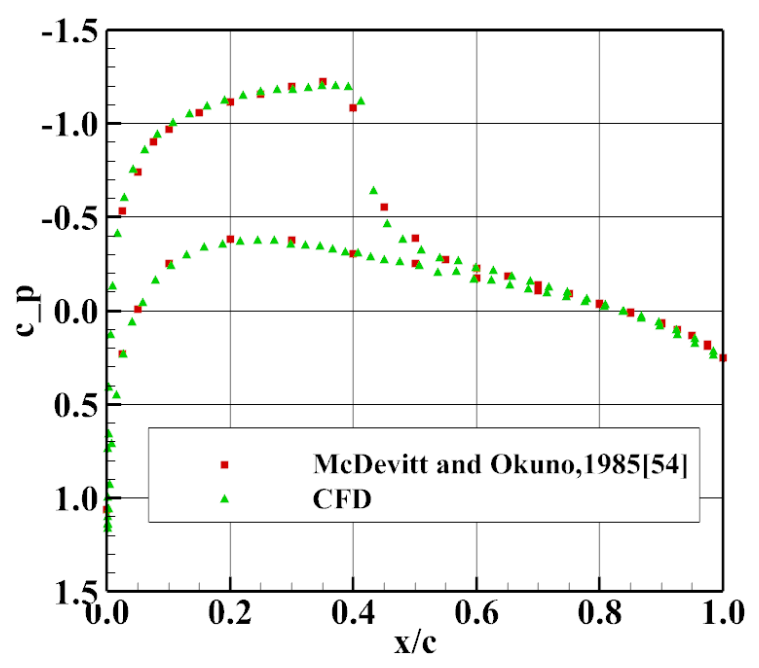

Figure 2. Comparison of numerical and test results.

To check out the unsteady flow simulation's capability of presenting numerical methods, an unsteady transonic flow around an NACA0012 airfoil was simulated at $\alpha=6^{\circ}, \mathrm{M}_{\infty}=0.751, \operatorname{Re}=1 \times 10^{7}$. The time history of lift coefficient is compared with that conducted by Raveh [56], as shown in Figure 3. The results indicate that the computed result matched well that from the published literature. Thus, the validated numerical methods and the grid parameters were adopted in the following research. 


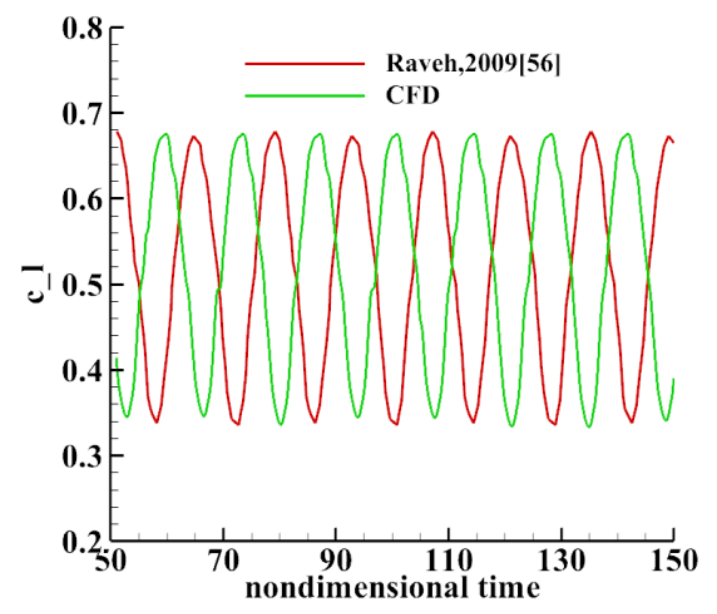

Figure 3. Comparison and validation of unsteady numerical results.

\section{Delay of Buffet Onset}

Under the same free-stream conditions of the validation case plotted in Figure 2, steady numerical simulations were conducted for the NACA0012 airfoil at different incidence angles. The calculated lift coefficients at different incidence angles are plotted, as shown in Figure 4. By referring to the work conducted by Iovnovich and Raveh [57] and Liu and Yang [26,27], the maximum curvature method proposed by Liu and Yang [26,27] is briefly introduced as follows.

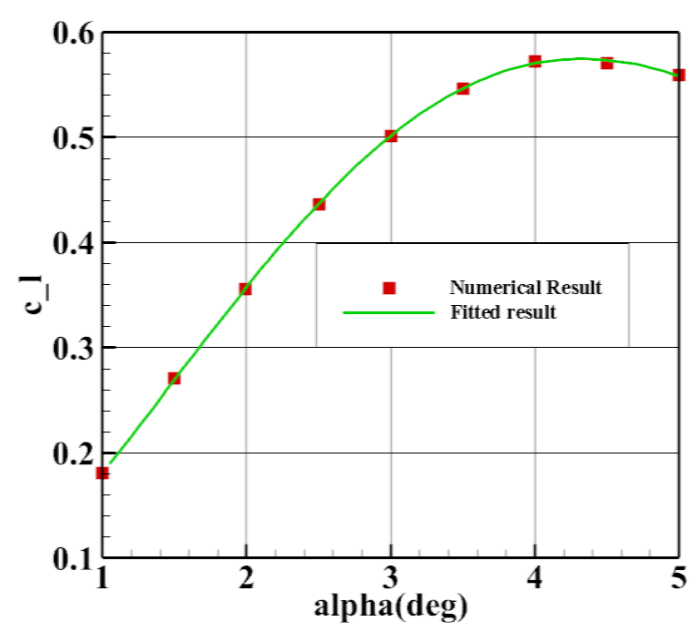

Figure 4. Lift coefficients vs. incidence angle.

By comparing the fitting precisions of polynomial aerodynamic curves, including lift and moment coefficient curves, it was found that a quartic polynomial can give the best fitting precision. It is written as follows:

$$
\mathrm{y}=a \mathrm{x}^{4}+b \mathrm{x}^{3}+c \mathrm{x}^{2}+d x+e
$$

where $\mathrm{a}, b, c, d$, and $e$ are fitting constants obtained by least-squares fitting, $x$ denotes the incidence angle, and y denotes either the lift or moment coefficient. In the present study, the lift coefficient was used.

The calculated curvature of $\mathrm{y}$ is as follows:

$$
\mathrm{K}(\mathrm{x})=\frac{\left|12 a \mathrm{x}^{2}+6 b x+2 c\right|}{\left(1+\left(4 a \mathrm{x}^{3}+3 b \mathrm{x}^{2}+3 c x+d\right)^{2}\right)^{3 / 2}}
$$


when the minimum curvature radius is achieved, the first-order derivative of $K(x)$ equals zero:

$$
\mathrm{K}^{\prime(\mathrm{x})}=0
$$

By solving Equation (3), the buffet onset incidence angle is obtained. The accuracy of this method is valid for NACA0012 airfoil buffet onset predictions, compared to wind tunnel tests, as validated in reference [26,27].

By using the above method, the curve plotted in Figure 4 was fitted by a quartic polynomial, and the maximum curvature was then obtained to be 0.8 , corresponding to a transonic buffet onset margin of $3.9^{\circ}$.

By inspecting the surface pressure distribution curve plotted in Figure 2, it can be found that the shockwave on the upper wing's surface resides in the range of $41 \% \sim 51 \%$ chord length; thus, $41 \%$ chord length, $46 \%$ chord length, and $51 \%$ chord length are denoted as " $41 \% \mathrm{x} / \mathrm{c}$ ", " $46 \% \mathrm{x} / \mathrm{c}$ ", and " $51 \%$ $\mathrm{x} / \mathrm{c}$ ", respectively. The tangential blowing position on the upper surface is first selected to be the " $46 \%$ $\mathrm{x} / \mathrm{c}^{\prime \prime}$ case. The geometric shape of the tangential blowing nozzle and the structured grid is plotted in Figure 5. This tangential blowing nozzle is a Venturi nozzle with its contraction ratio equaling 5.0. Its mass flow flux is governed and can be adjusted by setting the total pressure value at the inlet boundary of the Venturi nozzle.

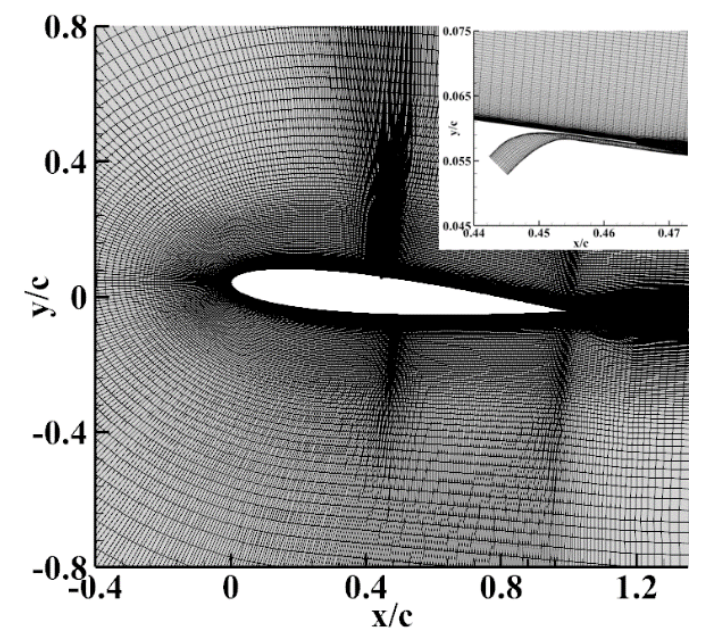

Figure 5. Grid of a tangential blowing slot.

With the blowing total pressure equaling the freestream total pressure, the lift coefficients at different incidence angles were compared with those of a clean airfoil, as plotted in Figure 6. Based on the maximum curvature method, the transonic buffet onset margin with " $46 \% \mathrm{x} / \mathrm{c}$ " blowing was found to be $4.5^{\circ}$, and its corresponding curvature was 0.04 . It can be seen from Figure 6 that, at Mach 0.751 when the incidence angle is below $2.5^{\circ}$, the airfoil lift with tangential blowing was a little lower. When the incidence angle is greater than $3.0^{\circ}$, and especially when the lift slope of the airfoil without blowing is lower for incidence angles beyond $4.5^{\circ}$, the airfoil lift with tangential blowing was higher. Thus, tangential blowing can not only postpone the transonic buffet onset margin, but the airfoil lift can also be increased. 


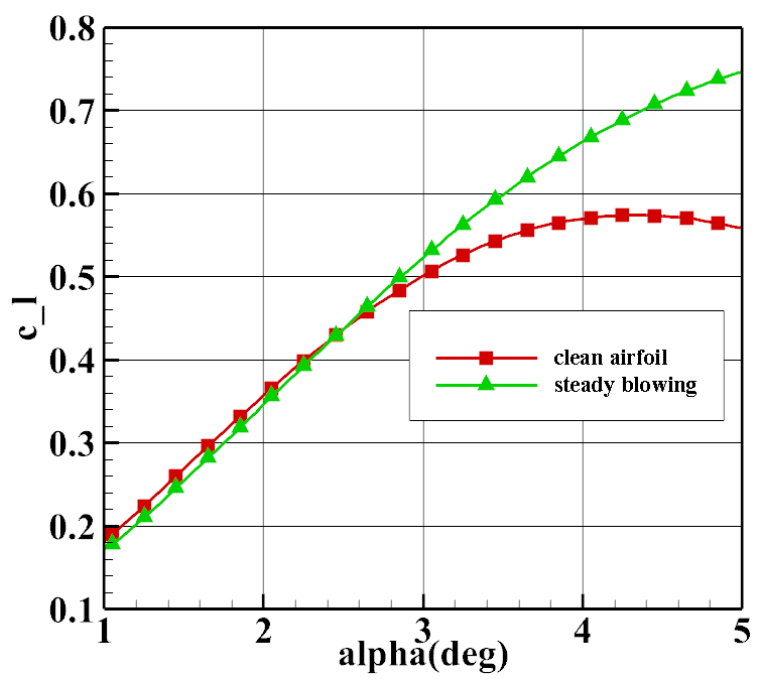

Figure 6. Comparison of lift coefficients with and without blowing.

To investigate the control effort, pressure coefficient distributions, pressure contours, and streamlines will be presented. At incidence angles of $4^{\circ}$ and $5^{\circ}$, the surface pressure coefficients of the airfoil with and without tangential slot blowing were plotted together, as shown in Figure 7. It is seen that the shock location is shifted downstream by tangential slot blowing. Besides, with the increase of incidence angle, the distance of the shockwave being pushed downstream will increase. The Mach number contours around airfoils with and without tangential slot blowing are plotted in Figure 8. It is found that the shockwave was sharpened and moved downstream by tangential slot blowing. Also, by comparing the streamline contours plotted in Figure 9 it is seen that there were two flow separation zones in the boundary layer $51 \% \mathrm{x} / \mathrm{c}$ for the clean airfoil (airfoil without blowing) case, which were induced by shockwave/boundary layer interactions and an adverse pressure gradient from the trailing edge, respectively. When tangential slot blowing was utilized, the size of the separation zone induced by the shockwave was reduced, and the other separation zone induced by the adverse pressure gradient disappeared, indicating stabilization of this control technology.

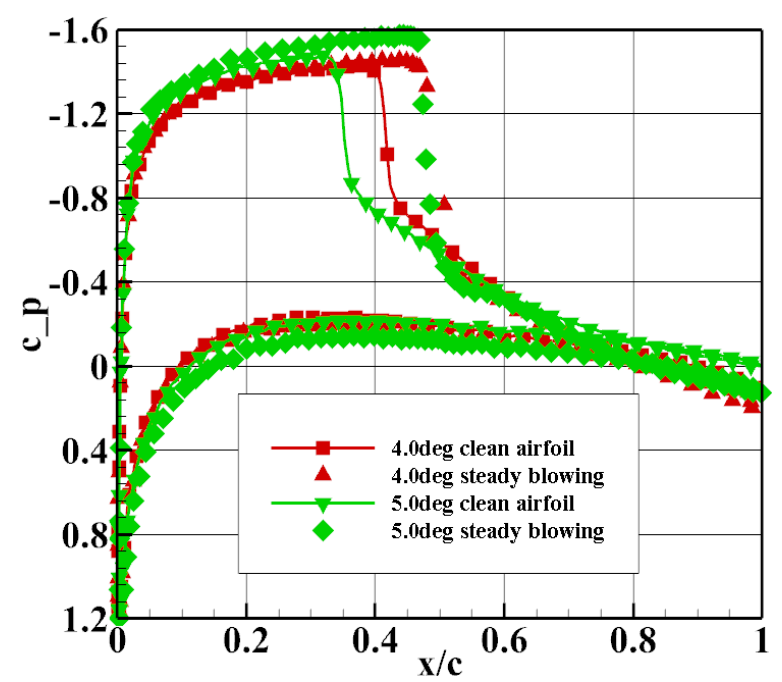

Figure 7. Surface pressure distribution with/without blowing. 


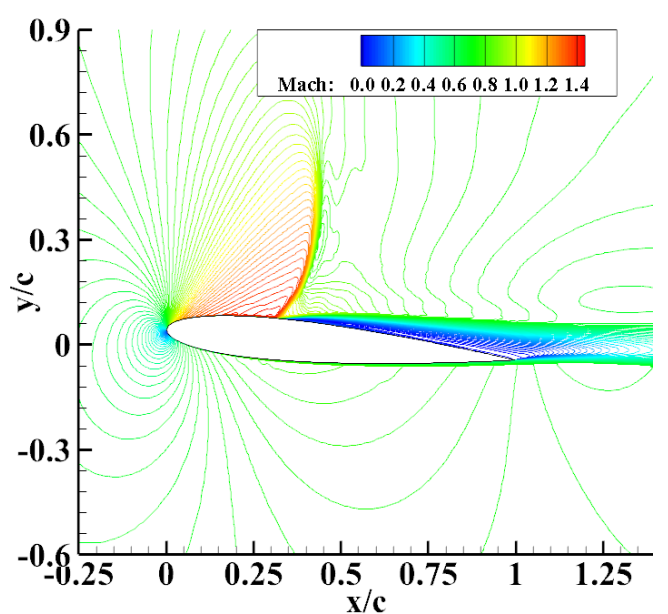

(a)

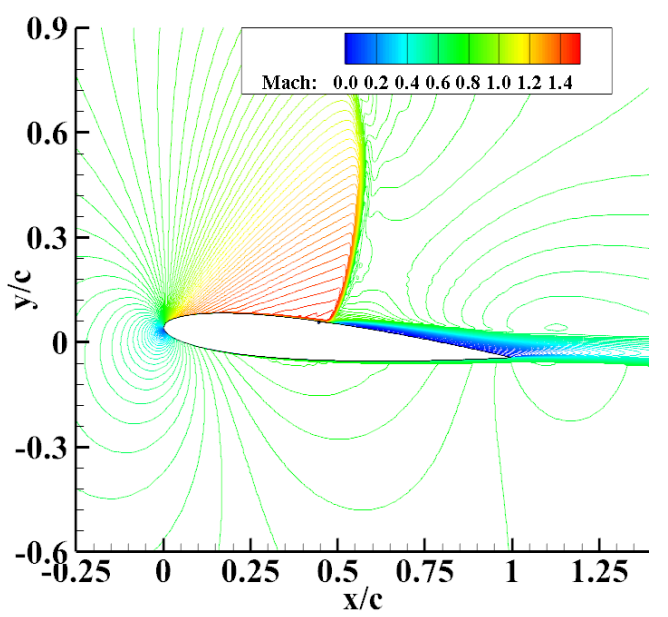

(b)

Figure 8. Comparison of unsteady numerical results (a) without tangential blowing and (b) with tangential slot blowing.

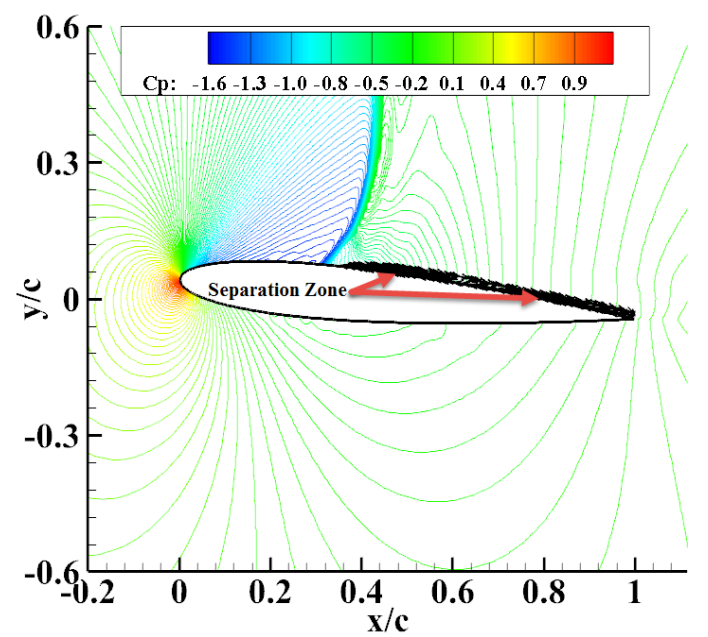

(a)

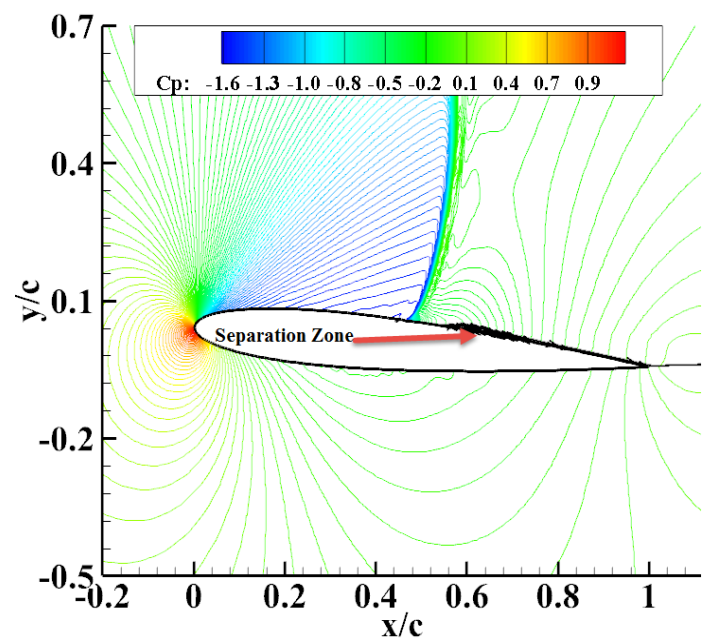

(b)

Figure 9. Streamlines at a $5.0^{\circ}$ incidence angle (a) without tangential blowing and (b) with tangential slot blowing.

\section{Alleviation of Buffet Load by Tangential Slot Blowing}

\subsection{Steady Blowing}

From the above context, the postponing of buffet onset margin is observed. In present section, the effect of tangential blowing on buffet load will be studies.

\subsubsection{Buffet Load Alleviation}

As with the buffeting margin, the load characteristics of the transonic buffet are also of great concern in the aviation industry. From the above context, it is seen that when the incidence angle exceeds $3.9^{\circ}$, transonic buffet will occur. At different buffeting states, namely at incidence angles of $5.0^{\circ}$, $5.5^{\circ}$, and $6.0^{\circ}$, the time histories of unsteady lift coefficients of the clean airfoil are plotted in Figure 10, and their corresponding PSD (power spectrum density) results are plotted together in Figure 11. From these two groups of figures, it is seen that with the increase of incidence angle, both the amplitude and peak frequency of the unsteady buffet load increased. Moreover, it should be noted that the peak frequency resides in the frequency range of $88-99 \mathrm{~Hz}$. In the wind-tunnel test of the NACA0012 airfoil given by McDevitt and Okuno [54], by referring to the flow parameters and the reduced frequency 
equation, the dimensional frequency was about $89 \mathrm{~Hz}$. Thus, the calculated dominant frequency agreed with that of the wind tunnel test.

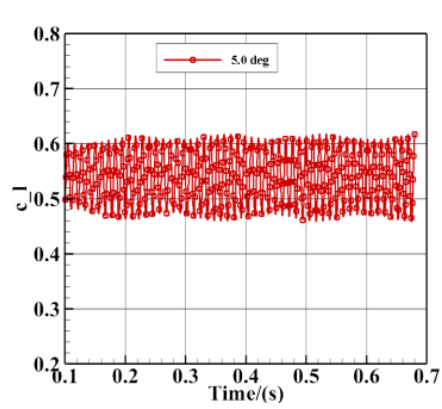

(a)

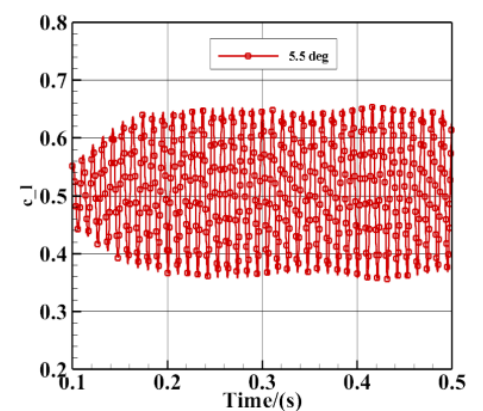

(b)

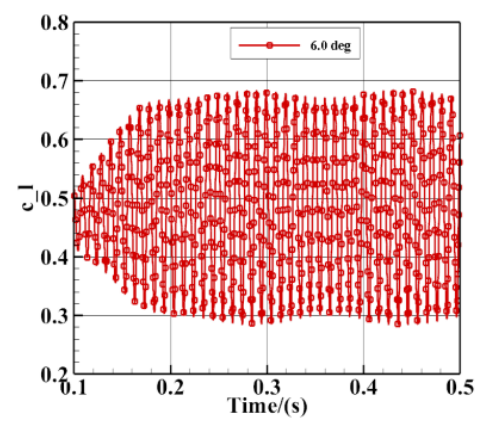

(c)

Figure 10. Unsteady lift coefficients at different incidence angles: (a) $5.0^{\circ}$, (b) $5.5^{\circ}$, and (c) $6.0^{\circ}$.

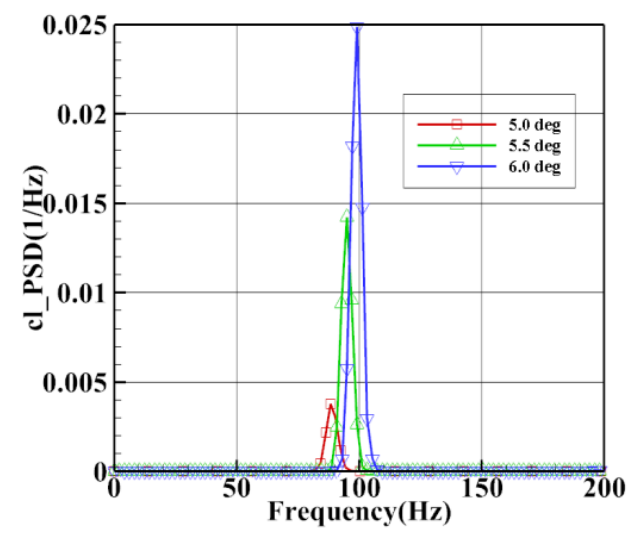

Figure 11. Power spectrum density (PSD) results at different incidence angles.

Accounting for the problem of transonic buffet load alleviation, 27 cases were simulated (i.e., flow fields under three different blowing locations (namely, the " $41 \% \mathrm{x} / \mathrm{c}$ " case, " $46 \% \mathrm{x} / \mathrm{c}$ " case, and " $51 \%$ $\mathrm{x} / \mathrm{c}^{\prime \prime}$ case) at three different incidence angles with three different blowing total pressures). In the present paper, "Blow_1.0_Ptot", "Blow_1.1_Ptot", and "Blow_1.2_Ptot" denote that the total pressure ratio of blowing to that of freestream flow is 1.0,1.1, and 1.2, respectively.

The time histories of unsteady airfoil lift coefficients of " $41 \% \mathrm{x} / \mathrm{c}$ " under different blowing pressure ratios at incidence angles $5.0^{\circ}, 5.5^{\circ}$, and $6.0^{\circ}$ are plotted in Figure 12. It is indicated that, for these incidence angles, the average airfoil lift increased and buffet loads decreased by using steady blowing with these three blow pressure ratios.

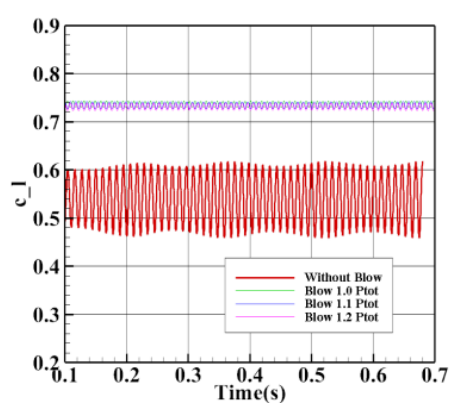

(a)

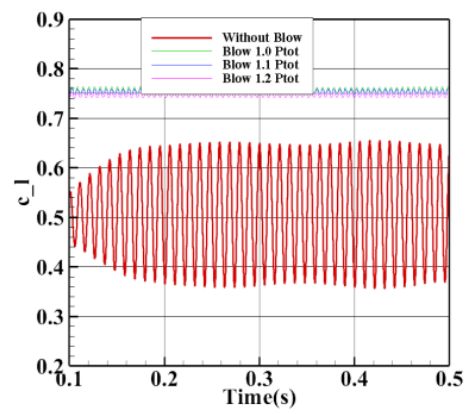

(b)

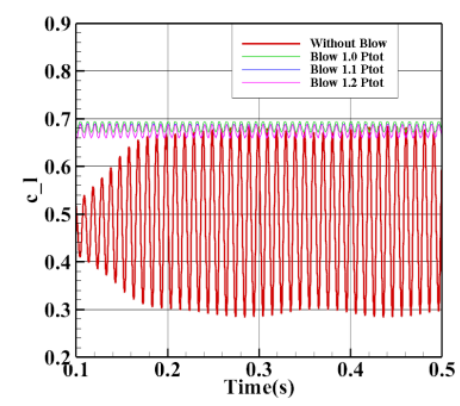

(c)

Figure 12. Unsteady lift coefficient histories under different total blowing pressures at different incidence angles: (a) $5.0^{\circ}$, (b) $5.5^{\circ}$, and (c) $6.0^{\circ}$. 
The PSD results of unsteady airfoil lift coefficients are plotted in Figure 13. It is shown that transonic buffet was suppressed by tangential slot blowing under the "Blow_1.0_Ptot" condition. With the increase of total pressure ratio from 1.0 to 1.1 and 1.2, the PSDs of unsteady airfoil lift coefficients were nearly the same, and buffet load alleviation was not apparently improved; thus, these two groups of PSD curves were not plotted.

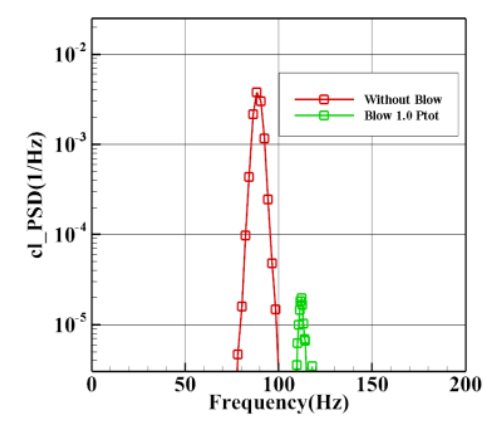

(a)

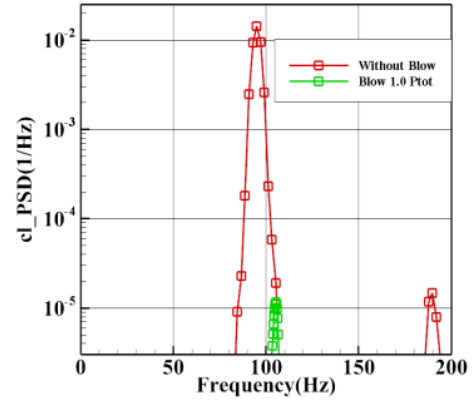

(b)

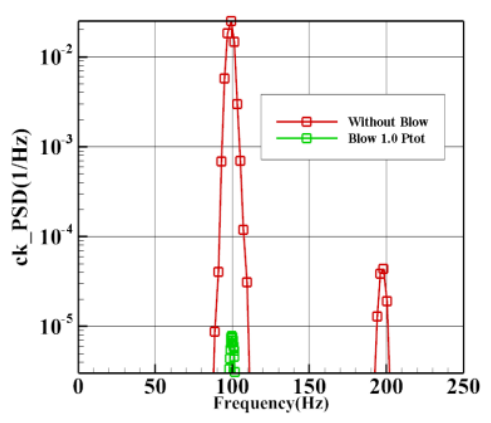

(c)

Figure 13. PSDs of unsteady lift coefficient histories under different blowing total pressures at different incidence angles: (a) $5.0^{\circ}$, (b) $5.5^{\circ}$, and (c) $6.0^{\circ}$.

With the blowing slot placed at $41 \% \mathrm{x} / \mathrm{c}$ chord location, the PSDs of airfoil lift coefficients at incidence angles $5.0^{\circ}, 5.5^{\circ}$, and $6.0^{\circ}$, corresponding to different blowing total pressure ratios, are plotted in Figure 14. It is seen that all these blowing cases with different blowing total pressure ratios can evidently depress buffet loads. For the " $5.0 \mathrm{deg}$ " case, an increase of blowing ratio could slightly improve the control effect, while for " $5.5 \mathrm{deg}$ " and " 6.0 deg" cases, their control effectiveness slightly deteriorated by increasing the total pressure ratio.

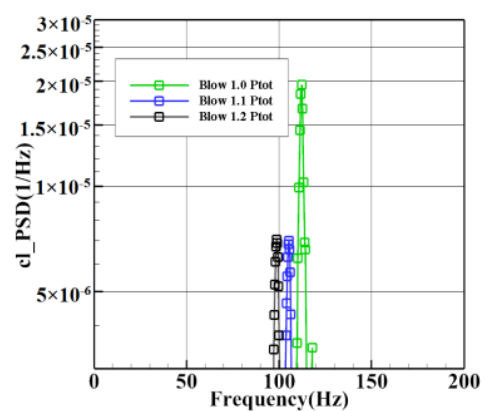

(a)

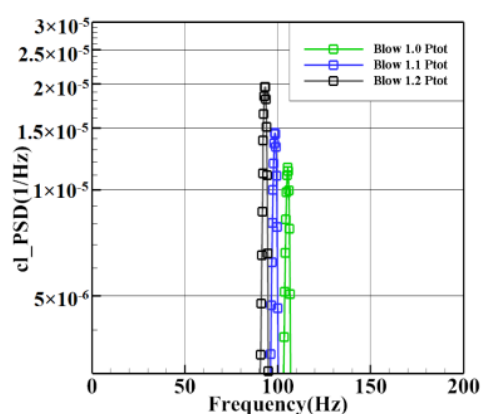

(b)

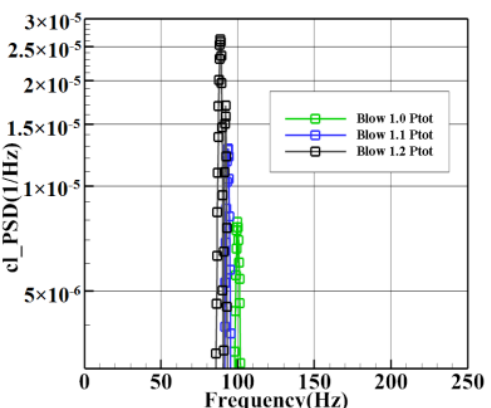

(c)

Figure 14. PSDs of unsteady lift coefficient histories under different blowing total pressures at different incidence angles: (a) $5.0^{\circ}$, (b) $5.5^{\circ}$, and (c) $6.0^{\circ}$.

The PSD results plotted in Figure 14 also suggest that, for all these blowing cases, the buffeting frequencies were nearly $100 \mathrm{~Hz}$, which indicates that the transonic buffet was alleviated, but the buffeting frequency was not evidently modified.

By comparing the PSD results of 27 cases, it is found that the influence of blowing conditions on the buffeting frequency is marginal, whereas the PSD peak increases at incidence angle $\alpha=5.5$ and 6.0 and experiences a small decrease at $\alpha=5.0$ as the blowing location moves from $41 \% \mathrm{x} / \mathrm{c}$ to $51 \% \mathrm{x} / \mathrm{c}$, which is evident in Table 1 . The results also show that the effectiveness of the blowing attains the peak at $41 \% \mathrm{x} / \mathrm{c}$, which suggests the blowing location is the key factor in buffeting control via steady blowing. 
Table 1. PSD peak values under the "Blow_1.0_Ptot" condition.

\begin{tabular}{cccc}
\hline & $\mathbf{5 . 0}^{\circ}$ & $\mathbf{5 . 5}^{\circ}$ & $\mathbf{6 . 0}^{\circ}$ \\
\hline $41 \% \mathrm{x} / \mathrm{c}$ & $1.96 \times 10^{-5}$ & $1.16 \times 10^{-5}$ & $7.86 \times 10^{-6}$ \\
\hline $46 \% \mathrm{x} / \mathrm{c}$ & $1.88 \times 10^{-5}$ & $1.36 \times 10^{-3}$ & $8.70 \times 10^{-3}$ \\
\hline $51 \% \mathrm{x} / \mathrm{c}$ & $1.58 \times 10^{-5}$ & $5.49 \times 10^{-3}$ & $1.63 \times 10^{-2}$ \\
\hline
\end{tabular}

\subsubsection{Flow Characteristics of the " $41 \% \mathrm{x} / \mathrm{c}$ " Case}

From the above context, it can be interpreted that flow characteristics are altered under the " $41 \%$ $\mathrm{x} / \mathrm{c}^{\prime \prime}$ case. The airfoil surface pressure coefficient distributions corresponding to maximum, median, and minimum lift states under "Blow_1.0_Ptot" tangential blowing are plotted in Figure 15. It is seen that shockwave location nearly did not oscillate, but surface pressure in the separation zone downstream of the shockwave varied with time.

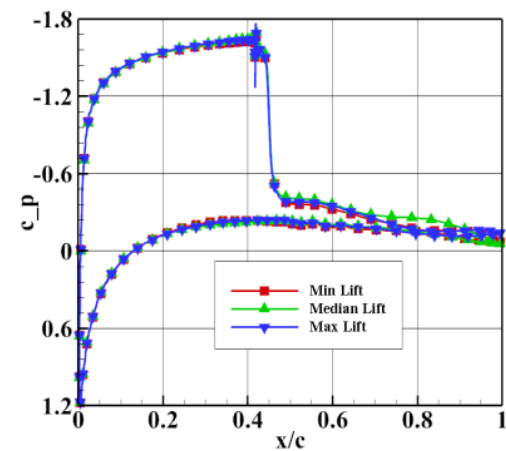

(a)

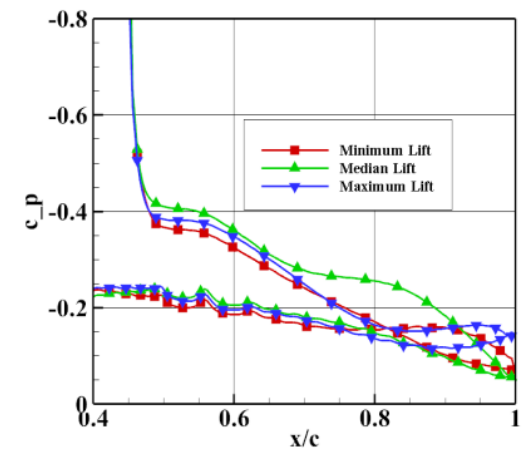

(b)

Figure 15. Surface pressure distributions corresponding to maximum/median/minimum lifts with tangential blowing. (a) Surface pressure distribution; (b) zoomed version.

Given that the most severe buffeting occurs at an incidence angle $6.0^{\circ}$, it was selected to highlight the control effort. Three streamline contours corresponding to maximum lift, mean lift, and minimum lift for clean airfoil and "Blow_1.0_Ptot" blowing cases are plotted in Figure 16. It is seen that, for the clean airfoil without tangential blowing, the aspects depicting the unsteady shock/separation bubble interaction intensity can be summarized as follows [57]: (a) wedge effects-pressure rise due to the shock resulted in flow separation emanating from the shock foot, where this separated region behaved similar to a geometric wedge to strengthen the shock, as shown in Figure 16a; (b) dynamic effects-as the shock moved upstream, the velocity of the shock increased the relative Mach number of the upstream flow, as shown in Figure 16b; (c) airfoil curvature effects-the expansion of flow through the subsonic zone was dependent on the local surface curvature. The thick boundary layer decreased the camber, resulting in a reduction of the airfoil's circulation, as shown in Figure 16c. Under tangential slot-blowing conditions plotted in Figure $16 \mathrm{~d}-\mathrm{f}$, by ejecting streamwise momentum into the boundary layer from tangential slot blowing, the adverse velocity of the separation bubble was evidently reduced, and the separation bubble could not move to the upstream location of the slot because of the strong ejection momentum. Hence, the shock/separation bubble interaction is evidently suppressed, resulting in (a) a reduced wedge effect as outlined as a smaller separation zone under the shock foot; (b) a weakened dynamic effect portrayed by the regularized bubble position. 


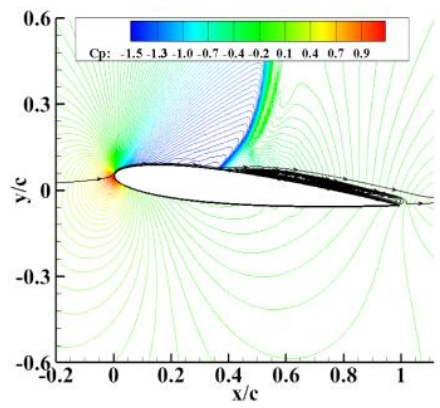

(a)

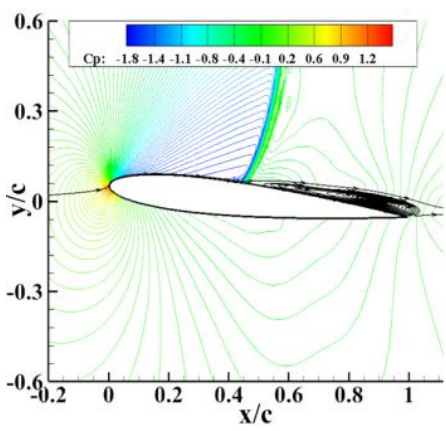

(d)

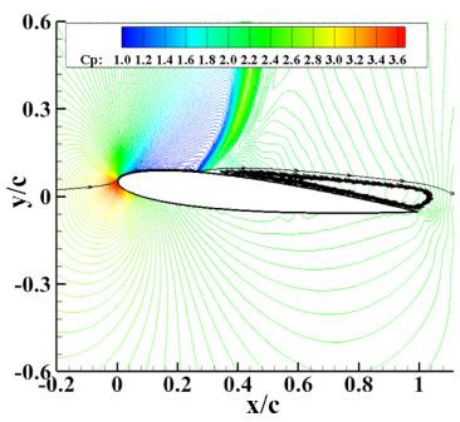

(b)

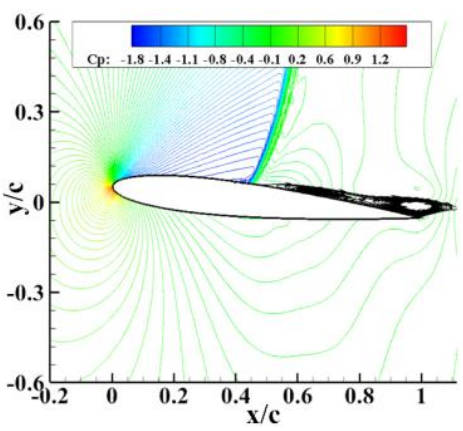

(e)

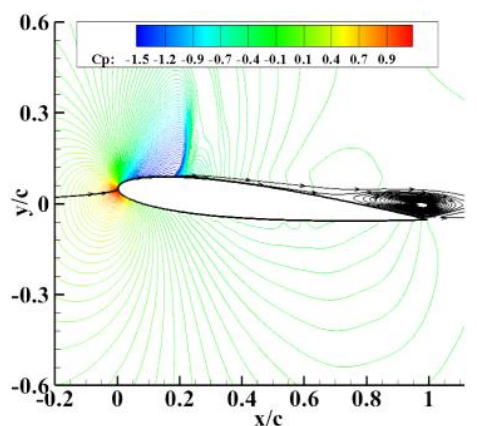

(c)

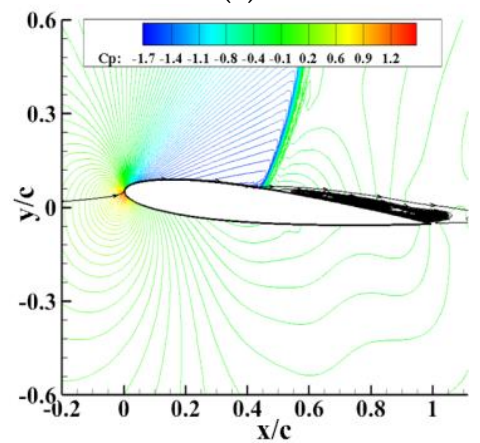

(f)

Figure 16. Streamlines corresponding to maximum/median/minimum lifts with and without tangential blowing. (a) Maximum lift without blowing; (b) median lift without blowing; (c) minimum lift without blowing; (d) maximum lift with blowing; (e) median lift with blowing; and (f) minimum lift with blowing.

To investigate the flow control mechanism, the Q-criterion results corresponding to those cases in Figure 16 were plotted in Figure 17, respectively. In the subsonic boundary layer, a feedback loop between the Kutta wave at the trailing edge and the location of the shock [58] was seen; the generated shear layer in the separation bubble under the shock foot warped pressure waves that propagated downstream, which interacted with the feedback of the Kutta waves travelling toward the shock from the trailing edge, completing the feedback loop. At the most upstream location, the shockwave shed its shear layer downstream and interacted with the shear layer under the shock foot as well as Kutta waves. These multiple interactions exchanged momentum and energy, sustaining oscillation of the periodic boundary layer, and unsteady buffet loads were generated, as shown in Figure 17a-f, the shear layer was generated by tangential slot blowing, and it continuously carried streamwise momentum. This shear layer prevented the upstream creeping of Kutta waves. Due to the curvature effect, the ejected shear layer uplifted the airfoil surface, and Kutta waves help this uplifting process to form a counter rotating eddy blob. Meanwhile, the shockwave did not shed its shear layer into separated flows, thus the interaction intensity was reduced. The momentum ejected from the blowing slot determines how far the reversed flow in boundary layer is influenced. With the increase of blowing total pressure, the feedback creeping was prevented more profoundly; thus, the buffet frequency slightly decreased. 


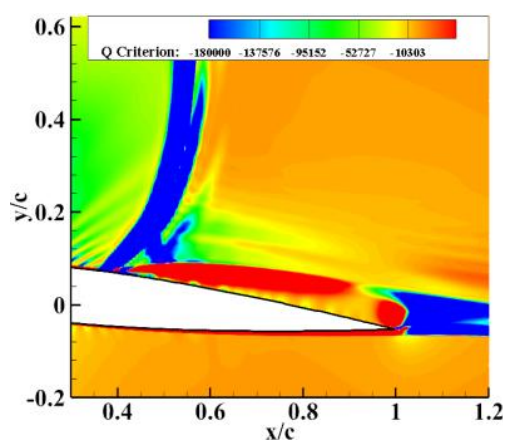

(a)

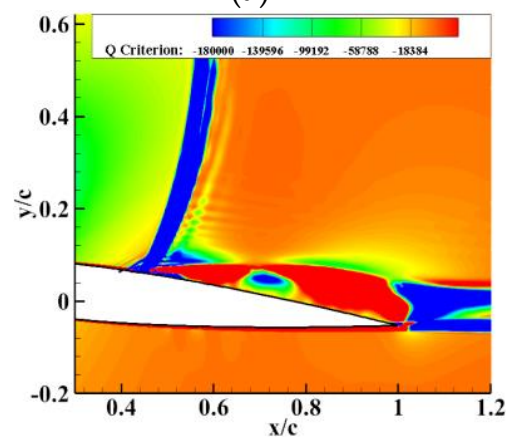

(d)

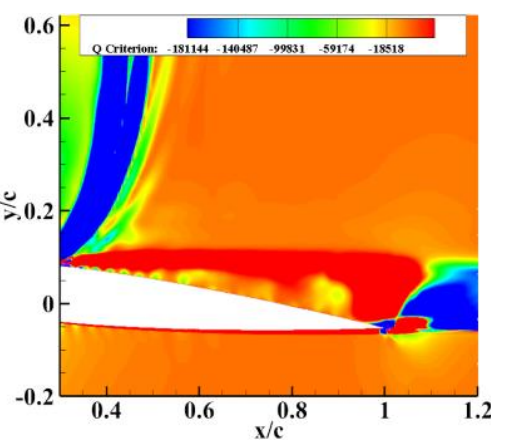

(b)

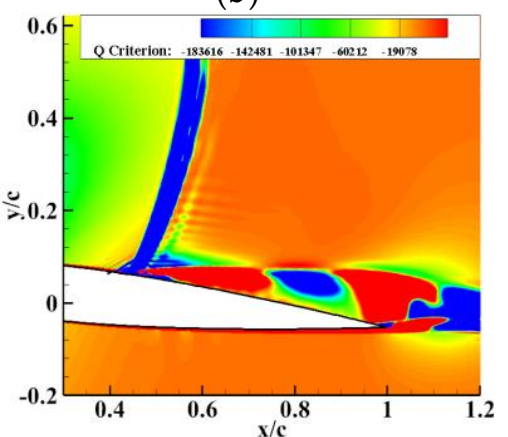

(e)

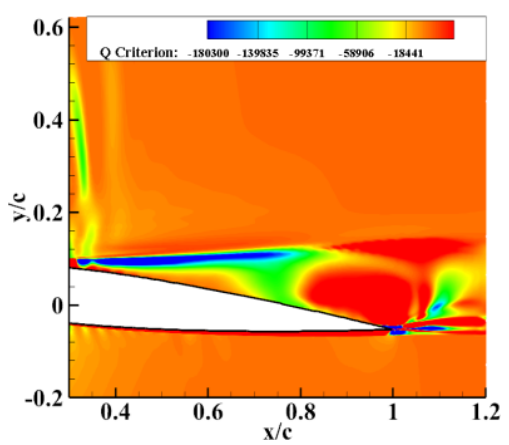

(c)

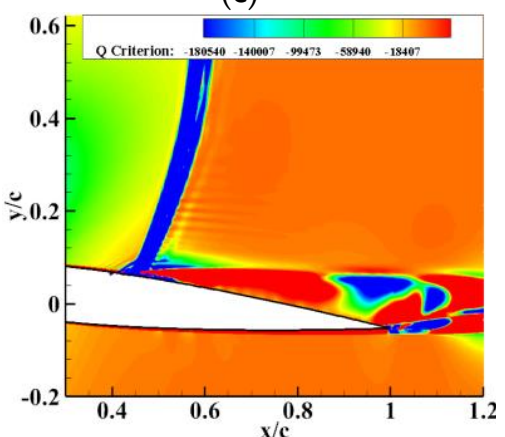

(f)

Figure 17. Q-criterion results corresponding to maximum/median/minimum lifts with and without tangential blowing. (a) Maximum lift without blowing; (b) median lift without blowing; (c) minimum lift without blowing; (d) maximum lift with blowing; (e) median lift with blowing; and (f) minimum lift with blowing.

\subsection{Periodic Blowing}

The effect of periodic tangential blowing was also investigated by transient numerical simulations. Given that the total pressure variation does not obviously influence the control effect of steady blowing cases, the variation ratio of total pressure was set to be 0.1 , and the periodic total pressure is depicted as

$$
P_{\text {tot_periodic }}=\left(1+\frac{\gamma-1}{2} M_{\infty}^{2}\right)^{\frac{\gamma}{\gamma-1}}+0.1 \times \sin (2 \pi \times f \times t) \times\left(1+\frac{\gamma-1}{2} M_{\infty}^{2}\right)^{\frac{\gamma}{\gamma-1}}
$$

By referring to the PSD results above, the frequency of periodic blowing was set to be $90 \mathrm{~Hz}$, and $\gamma$ was 1.4 for calorically perfect gas.

From the above context, it is known that the most effective effect was achieved when the tangential blowing slot was placed at the location denoted as " $41 \% \mathrm{x} / \mathrm{c}$ ". Thus, the periodic blowing slot was first placed here. The unsteady lift coefficient results under periodic blowing conditions at different incidence angles were compared with those of steady blowing cases, as shown in Figure 18. By examining the results, it is found that the unsteady lift coefficients exhibited an oscillating frequency nearly the same as that of the clean airfoil at incidence angles $5.0^{\circ}, 5.5^{\circ}$, and $6.0^{\circ}$. However, the mean lift coefficient values under periodic blowing conditions were a little lower compared to their corresponding steady blowing cases, as presented in Table 2.

Table 2. Mean lift coefficients with and without blowing.

\begin{tabular}{clll}
\hline & $\mathbf{5 . 0}^{\circ}$ & $\mathbf{5 . 5}^{\circ}$ & $\mathbf{6 . 0}^{\circ}$ \\
\hline Steady blowing & 0.73 & 0.75 & 0.71 \\
Periodic blowing & 0.71 & 0.70 & 0.65 \\
\hline
\end{tabular}




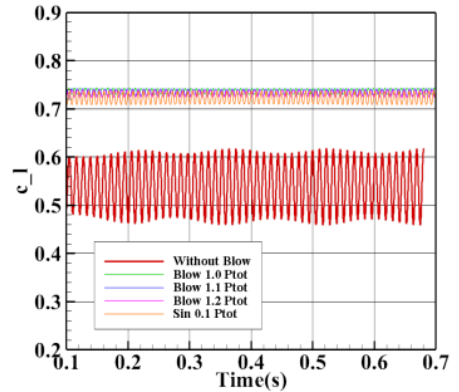

(a)

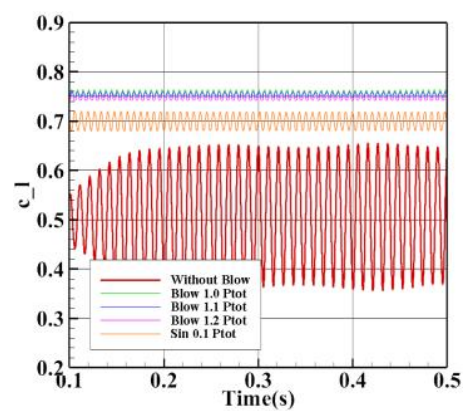

(b)

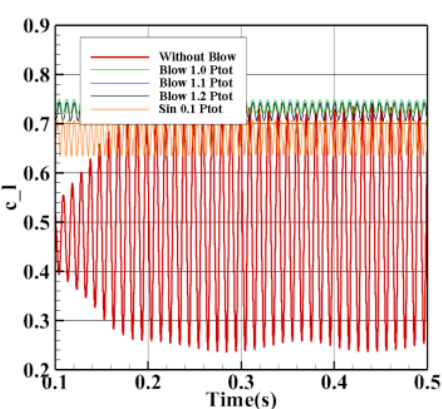

(c)

Figure 18. Unsteady lift coefficient histories under the " $41 \% \mathrm{x} / \mathrm{c}^{\prime}$ c case at: (a) $5.0^{\circ}$, (b) $5.5^{\circ}$, and (c) $6.0^{\circ}$.

To investigate the frequency characteristics of unsteady lift coefficients, the PSD results of different periodic blowing cases were plotted, as shown in Figure 19. It can be seen from these figures that, for the " $41 \% \times / \mathrm{c}$ " case, the peak PSD value of the lift coefficient with periodic tangential blowing was about 5 times larger than that with steady tangential blowing. Moreover, with the increase of incidence angle, frequencies corresponding to the peak PSD values also increased. Compared to the PSD results for steady blowing cases plotted in Figure 14, there was no obvious difference in frequency characteristics. Under all three blowing locations, periodic tangential slot blowing gave higher peak PSD values compared to steady tangential slot blowing at those two blowing locations.

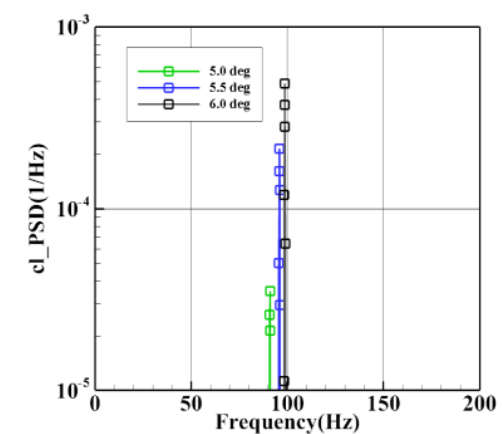

(a)

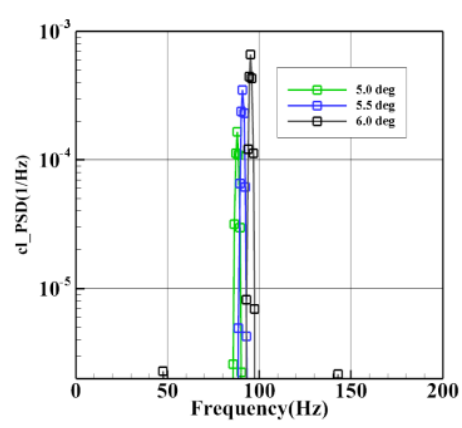

(b)

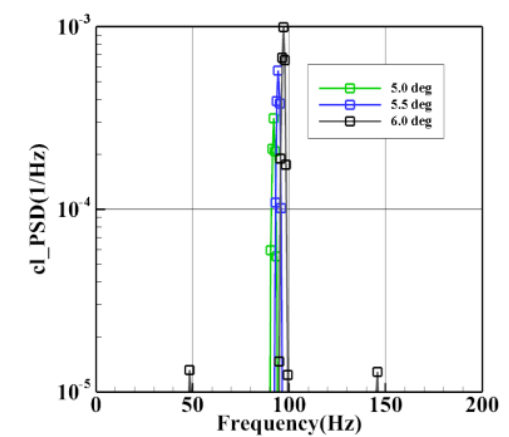

(c)

Figure 19. PSDs of unsteady lift coefficient histories under different blowing cases: (a) $41 \% \mathrm{x} / \mathrm{c}$, (b) $46 \%$ $\mathrm{x} / \mathrm{c}$, and (c) $51 \% \mathrm{x} / \mathrm{c}$.

Surface pressure coefficient distributions are plotted in Figures 20-22. Within each figure, surface pressure distribution lines corresponding to maximum, median, and minimum lifts in one oscillating period are plotted together. From these figures, it is observed that these oscillation amplitudes were smaller compared to those without tangential blowing (plotted in Figure 7), while compared to the results of steady tangential blowing cases (plotted in Figure 15), the shock oscillation amplitudes were a little larger, while the surface pressure variation downstream of the shockwave was evidently smaller. 


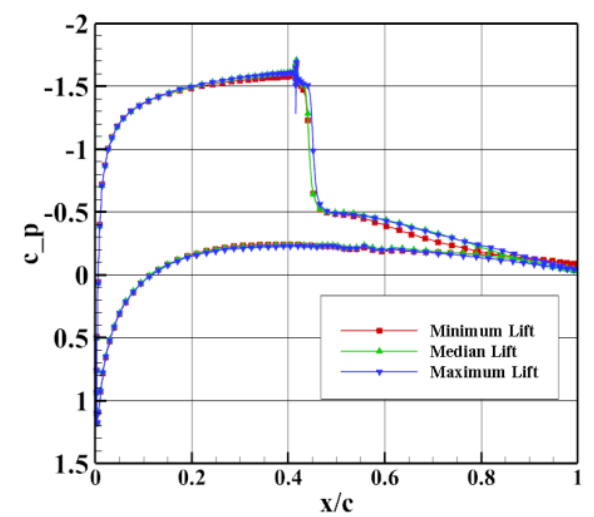

(a)

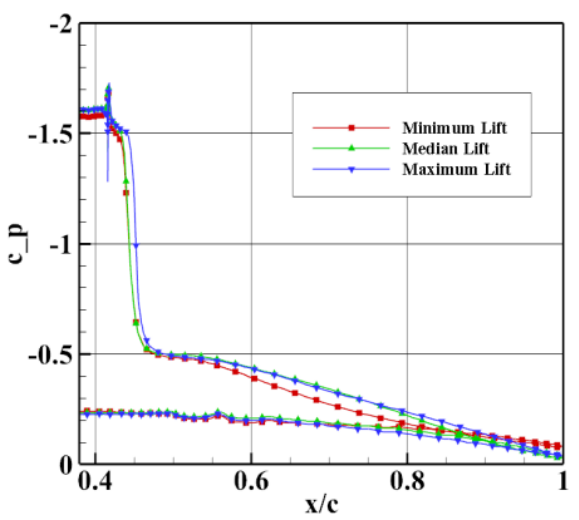

(b)

Figure 20. Pressure distributions over the airfoil for the " $41 \% \mathrm{x} / \mathrm{c}$ " case at an incidence angle of $6.0^{\circ}$. (a) Surface pressure distribution; (b) zoomed version.

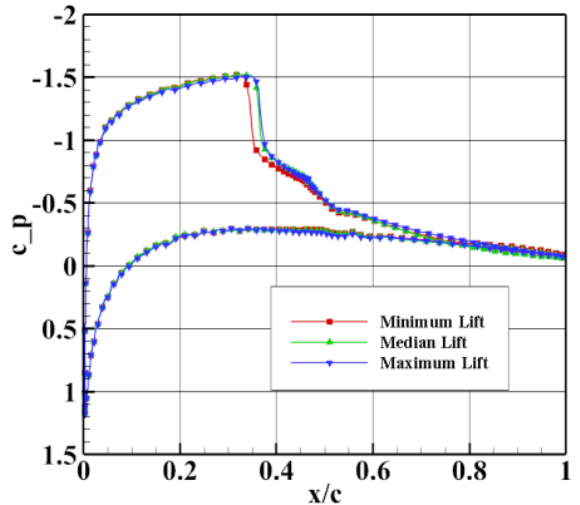

(a)

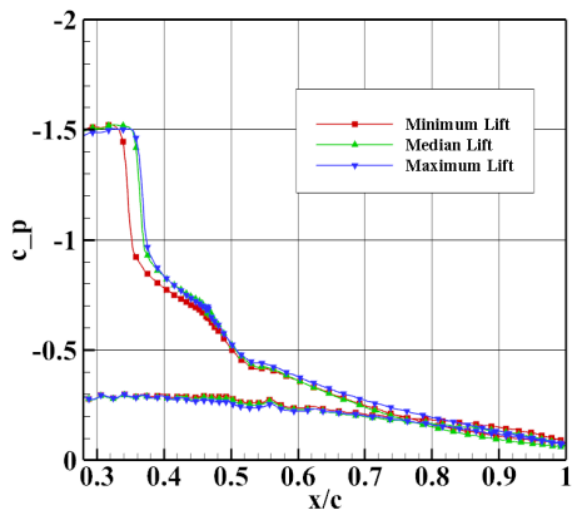

(b)

Figure 21. Pressure distributions over the airfoil for the " $46 \% \mathrm{x} / \mathrm{c}^{\prime \prime}$ case at an incidence angle of $6.0^{\circ}$. (a) Surface pressure distribution; (b) zoomed version.

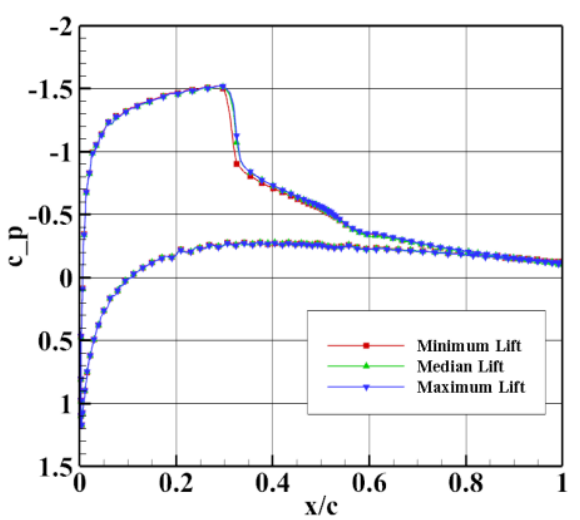

(a)

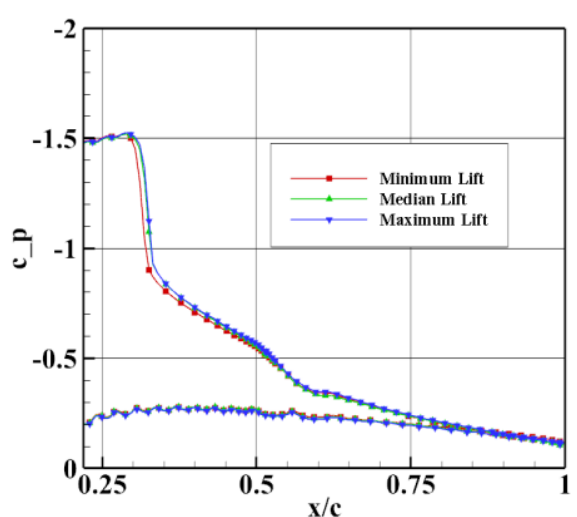

(b)

Figure 22. Pressure distributions over the airfoil for the " $51 \% \mathrm{x} / \mathrm{c}$ " case at an incidence angle of $6.0^{\circ}$. (a) Surface pressure distribution; (b) zoomed version.

Q-criterion results corresponding to minimum lift, median lift, and maximum lift, are plotted in Figures 23-25, respectively. It can be seen from these figures that the blowing slots were all downstream of the shock foot. For the " $41 \% \mathrm{x} / \mathrm{c}$ " case, the control mechanism was similar to that stated above for steady blowing cases. The difference is that the ejected momentum from periodic blowing was smaller; thus, Kutta waves can creep more upstream comparing to steady blowing cases, inducing a relatively larger buffet load. As the blowing slot moved downstream, the distance between shock and blowing 
slot increased. Although the ejected momentum could also reduce the reverse boundary layer velocity to suppress Kutta waves on the upper surface of airfoil, its control effect on the separation bubble was obviously weakened; thus, the buffet load increased as the blowing slot moved downstream.

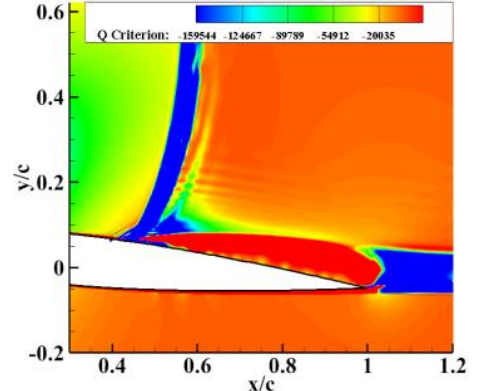

(a)

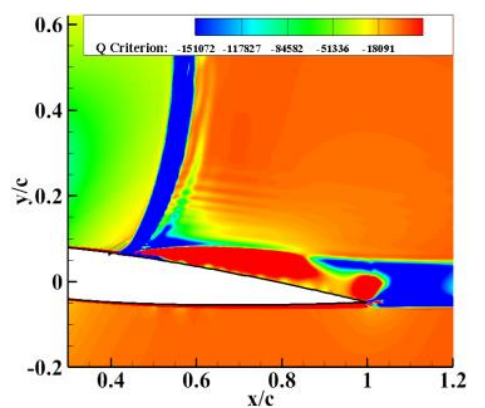

(b)

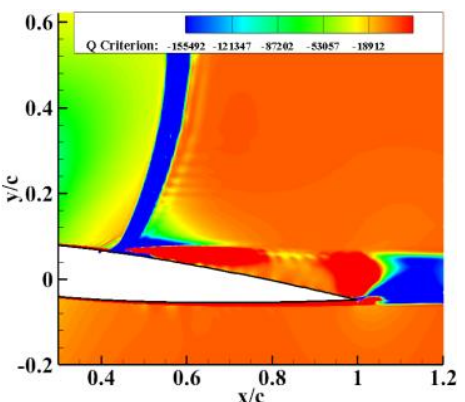

(c)

Figure 23. Q-criterion results for the " $41 \% \mathrm{x} / \mathrm{c}$ " case. (a) Maximum lift; (b) median lift; and (c) minimum lift.

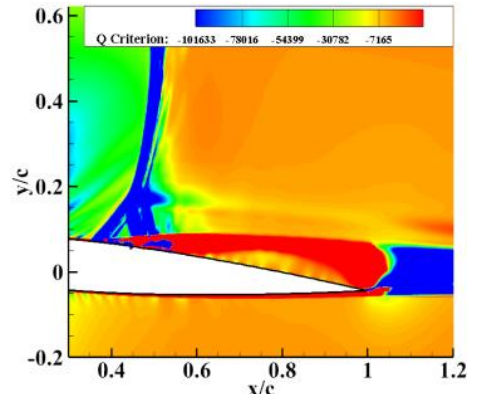

(a)

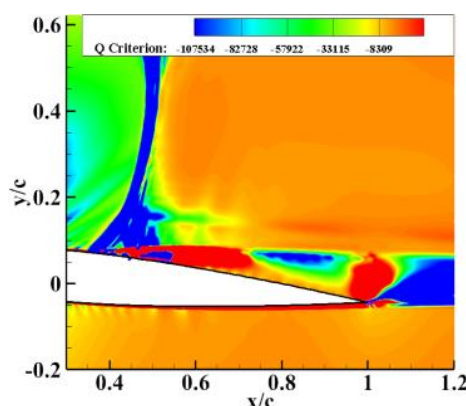

(b)

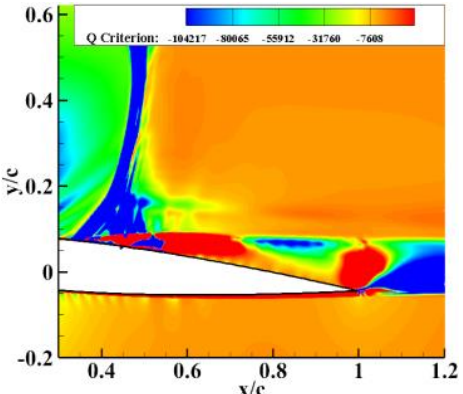

(c)

Figure 24. Q-criterion results for the " $46 \% \mathrm{x} / \mathrm{c}$ " case. (a) Maximum lift; (b) median lift; and (c) minimum lift.

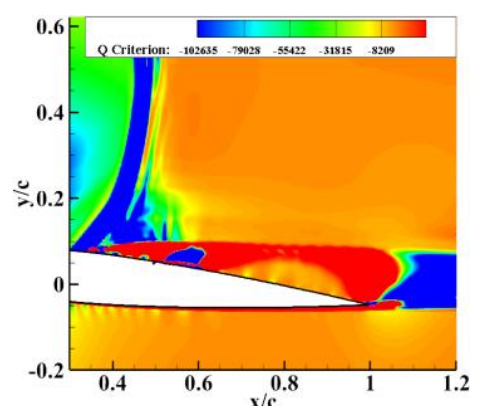

(a)

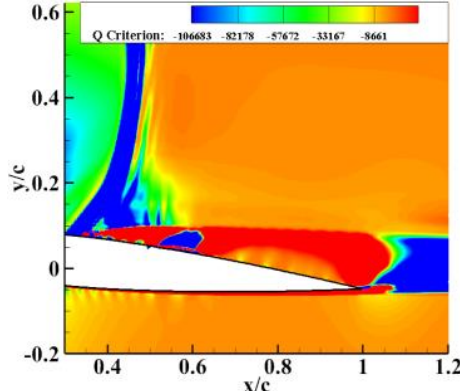

(b)

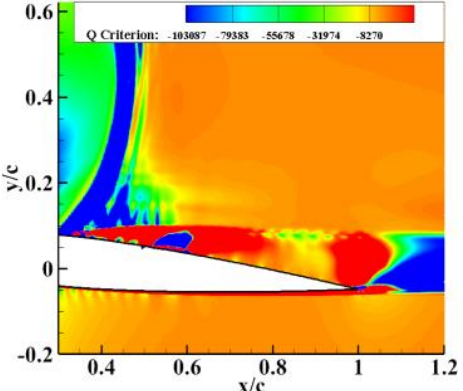

(c)

Figure 25. Q-criterion results for the "51\% x/c" case. (a) Maximum lift; (b) median lift; and (c) minimum lift.

With the increase of incidence angle, the separation bubble increased, inducing increases in interference intensity, feedback control, and a decrease in the interference zone size. This induces the increase of buffet frequency with incidence angle.

\section{Conclusions}

By using a Venturi nozzle over the upper surface of an airfoil, the control effects of tangential slot blowing on postponement of the transonic buffet onset margin and alleviation of buffet load were investigated numerically. It was found that steady tangential blowing could delay buffet onset, and an 
evident increase of the lift coefficient was discerned at incidence angles near and exceeding the buffet onset margin of the clean airfoil. In the investigation of buffet load alleviation, both steady and periodic tangential blowing with slots placed at three different chord-wise locations were tested under buffeting states of a clean airfoil. The results indicated that unsteady buffet loads could be greatly suppressed by both steady and periodic blowing. An increase in total blowing pressure does not apparently improve the reduction effect of dynamic loads for steady blowing cases; the control effect is reduced as the blowing slot moves downstream. The control effort is as follows: the shock/separation bubble interaction is evidently suppressed, resulting in (a) a reduced wedge effect as outlined as a smaller separation zone under the shock foot; (b) a weakened dynamic effect portrayed by the regularized bubble position. The buffet mechanism includes (a) the feedback loop between the Kutta wave and the separation bubble under the shock foot, and (b) the interaction between the shear layer shed by the shockwave and Kutta waves. Under blowing conditions, the upstream creeping Kutta waves are prevented, and the intensity of the shear layer shed by the shockwave into separated flows is evidently reduced. Parametric studies show that, with the increase of total blowing pressure for steady blowing cases, or with the increase of incidence angle for periodic blowing cases, the feedback distance decreases; thus, the buffet frequency increases. The mean lifts of steady cases are slightly higher compared to their corresponding periodic blowing cases. Steady blowing at a $41 \mathrm{x} / \mathrm{c}$ chord location is suggested among the presented test cases.

Author Contributions: investigation, H.D. (Huixue Dang); methodology, J.Z. and Z.Y.; writing and editing, H.D. (Huibo Dang).

Funding: This research was funded by National Natural Science Foundation of China, grant number 51308519 and 11472216 .

Conflicts of Interest: The funders had no role in the design of the study; in the collection, analyses, or interpretation of data; in the writing of the manuscript; or in the decision to publish the results.

\section{References}

1. Huang, J.; Xiao, Z.; Liu, J.; Fu, S. Simulation of shock wave buffet and its suppression on an OAT15A supercritical airfoil by IDDES. China Phys. Mech. 2012, 55, 260-271. [CrossRef]

2. Giannelis, N.F.; Vio, G.A.; Levinski, O. A review of recent developments in the understanding of transonic shock buffet. Prog. Aerosp. Sci. 2017, 92, 39-84. [CrossRef]

3. Szubert, D.; Grossi, F.; Jimenez Garcia, A.; Yannick, H.; Julian, C.R.H.; Marianna, B. Shock-vortex shear-layer interaction in the transonic flow around a supercritical airfoil at high Reynolds number in buffet conditions. J. Fluid Struct. 2015, 55, 276-302. [CrossRef]

4. Timme, S.; Thormann, R. Towards Three-Dimensional Global Stability Analysis of Transonic Shock Buffet. In Proceedings of the AIAA Atmospheric Flight Mechanics Conference, American Institute of Aeronautics and Astronautics, Washington, DC, USA, 13-17 June 2016. [CrossRef]

5. Zhang, Z.; Qu, K. Numerical Investigation of Transonic Airfoil Buffet Suppression. In Proceedings of the 53rd AIAA Aerospace Sciences Meeting, American Institute of Aeronautics and Astronautics, Kissimmee, FL, USA, 5-9 January 2015. [CrossRef]

6. Ohmichi, Y.; Ishida, T.; Hashimoto, A. Numerical Investigation of Transonic Buffet on a Three-Dimensional Wing using Incremental Mode Decomposition. In Proceedings of the 55th AIAA Aerospace Sciences Meeting, American Institute of Aeronautics and Astronautics, Grapevine, TX, USA, 9-13 January 2017. [CrossRef]

7. Dandois, J.; Lepage, A.; Dor, J.B.; Molton, P.; Ternoy, F.; Geeraert, A.; Brunet, V.; Coustols, É. Open and closed-loop control of transonic buffet on 3D turbulent wings using fluidic devices. C. R. Mécanique 2014, 342, 425-436. [CrossRef]

8. Sartor, F.; Mettot, C.; Sipp, D. Stability, Receptivity, and Sensitivity Analyses of Buffeting Transonic Flow over a Profile. AIAA J. 2014, 53, 1980-1993. [CrossRef]

9. Gao, C.; Zhang, W.; Ye, Z. Numerical study on closed-loop control of transonic buffet suppression by trailing edge flap. Comput. Fluids 2016, 132, 32-45. [CrossRef]

10. Tian, Y.; Liu, P.; Li, Z. Multi-objective optimization of shock control bump on a supercritical wing. Sci. China (Technol. Sci.) 2014, 1, 192-202. [CrossRef] 
11. Ishida, T.; Ishiko, K.; Hashimoto, A.; Aoyama, T.; Takekawa, K. Transonic Buffet Simulation over Supercritical Airfoil by Unsteady-FaSTAR Code. In Proceedings of the 54th AIAA Aerospace Sciences Meeting, American Institute of Aeronautics and Astronautics, San Diego, CA, USA, 4-8 January 2016. [CrossRef]

12. Fukushima, Y.; Kawai, S. Wall-modeled Large-Eddy Simulation of Transonic Buffet over a Supercritical Airfoil at high Reynolds Number. In Proceedings of the 55th AIAA Aerospace Sciences Meeting, American Institute of Aeronautics and Astronautics, Grapevine, TX, USA, 9-13 January 2017. [CrossRef]

13. Raveh, D.E.; Dowell, E.H. Aeroelastic Responses of Elastically Suspended Airfoil Systems in Transonic Buffeting Flows. AIAA J. 2014, 52, 926-934. [CrossRef]

14. Tian, Y.; Li, Z.; Liu, P.Q. Upper Trailing-Edge Flap for Transonic Buffet Control. J. Aircr. 2017, 1, 8. [CrossRef]

15. Quan, J.; Zhang, W.; Gao, C.; Ye, Z. Characteristic analysis of lock-in for an elastically suspended airfoil in transonic buffet flow. Chin. J. Aeronaut. 2016, 29, 129-143. [CrossRef]

16. Tian, Y.; Feng, P.; Liu, P.; Hu, T.; Qu, Q. Spoiler Upward Deflection on Transonic Buffet Control of Supercritical Airfoil and Wing. J. Aircr. 2016, 54, 1229-1233. [CrossRef]

17. Crouch, J.D.; Garbaruk, A.; Magidov, D. Predicting the onset of flow unsteadiness based on global instability. J. Comput. Phys. 2007, 224, 924-940. [CrossRef]

18. Dietz, G.; Schewe, G.; Mai, H. Amplification and amplitude limitation of heave/pitch limit-cycle oscillations close to the transonic dip. J. Fluid Struct. 2006, 22, 505-527. [CrossRef]

19. Lee, B.H.K. Self-sustained shock oscillations on airfoils at transonic speeds. Prog. Aerosp. Sci. 2001, 37, 147-196. [CrossRef]

20. Hermes, V.; Klioutchnikov, I.; Olivier, H. Numerical investigation of unsteady wave phenomena for transonic airfoil flow. Aerosp. Sci. Technol. 2013, 25, 224-233. [CrossRef]

21. Bouhadji, A.; Braza, M. Organised modes and shock-vortex interaction in unsteady viscous transonic flows around an aerofoil: Part I: Mach number effect. Comput. Fluids 2003, 32, 1233-1260. [CrossRef]

22. Bouhadji, A.; Braza, M. Organised modes and shock-vortex interaction in unsteady viscous transonic flows around an aerofoil: Part II: Reynolds number effect. Comput. Fluids 2003, 32, 1261-1281. [CrossRef]

23. Caruana, D.; Mignosi, A.; Corrège, M.; Le Pourhiet, A.; Rodde, A.M. Buffet and buffeting control in transonic flow. Aerosp. Sci. Technol. 2005, 9, 605-616. [CrossRef]

24. Tian, Y.; Gao, S.; Liu, P.; Wang, J. Transonic buffet control research with two types of shock control bump based on RAE2822 airfoil. Chin. J. Aeronaut. 2017, 30, 1681-1696. [CrossRef]

25. Murman, S.M.; Diosady, L.T.; Blonigan, P.J. Comparison of Transonic Buffet Simulations with Unsteady PSP Measurements for a Hammerhead Payload Fairing. In Proceedings of the 55th AIAA Aerospace Sciences Meeting, American Institute of Aeronautics and Astronautics, Grapevine, TX, USA, 9-13 January 2017. [CrossRef]

26. Liu, J.; Yang, Z. A Maximum Curvature Method for Predicting Transonic Buffet Onset Based on Steady Aerodynamic Parameters. Procedia Eng. 2013, 61, 363-369. [CrossRef]

27. Yang, Z.; Liu, J. A Precise Approach to Predict Transonic Wing Buffet Boundary. Mech. Sci. Technol. Aerosp. Eng. 2013, 12, 1717-1721. (In Chinese)

28. Kou, J.; Zhang, W. An improved criterion to select dominant modes from dynamic mode decomposition. Eur. J. Mech. B Fluids 2017, 62, 109-129. [CrossRef]

29. Barakos, G.; Drikakis, D. Numerical simulation of transonic buffet flows using various turbulence closures. Int. J. Heat Fluid Flow 2000, 21, 620-626. [CrossRef]

30. Thiery, M.; Coustols, E. Numerical prediction of shock induced oscillations over a 2D airfoil: Influence of turbulence modelling and test section walls. Int. J. Heat Fluid Flow 2006, 27, 661-670. [CrossRef]

31. Bonnifet, V.; Gerolymos, G.A.; Vallet, I. Transonic Buffet Prediction using Partially Averaged Navier-Stokes. In Proceedings of the 23rd AIAA Computational FLUID DYNAMICS Conference, American Institute of Aeronautics and Astronautics, Denver, CO, USA, 5-9 June 2017. [CrossRef]

32. Iovnovich, M.; Raveh, D.E. Transonic unsteady aerodynamics in the vicinity of shock-buffet instability. J. Fluid Struct. 2012, 29, 131-142. [CrossRef]

33. Thiede, P.K.P.; Stanewsky, E. Active and Passive Shock/Boundary Layer Interaction Control on Supercritical Airfoils; AGARD CP-365, DEUTSCHE FORSCHUNGS-UND VERSUCHSANSTALT FUER LUFT-UND RAUMFAHRT EV GOETTINGEN (GERMANY FR); Reston: Brussels, Belgium, 1984.

34. Smith, A.; Babinsky, H.; Fulker, J.L.; Ashill, P.R. Shock Wave/ Boundary-Layer Interaction Control Using Streamwise Slots in Transonic Flows. J. Aircr. 2004, 41, 540-546. [CrossRef] 
35. Holden, H.A.; Babinsky, H. Separated Shock-Boundary-Layer Interaction Control Using Streamwise Slots. J. Aircr. 2005, 42, 166-171. [CrossRef]

36. Molton, P.; Dandois, J.; Lepage, A.; Brunet, V.; Bur, R. Control of Buffet Phenomenon on a Transonic Swept Wing. AIAA J. 2013, 51, 761-772. [CrossRef]

37. Ogawa, H.; Babinsky, H.; Pätzold, M.; Lutz, T. Shock-Wave/Boundary-Layer Interaction Control Using Three-Dimensional Bumps for Transonic Wings. AIAA J. 2008, 46, 1442-1452. [CrossRef]

38. Eastwood, J.P.; Jarrett, J.P. Toward Designing with Three-Dimensional Bumps for Lift/Drag Improvement and Buffet Alleviation. AIAA J. 2012, 50, 2882-2898. [CrossRef]

39. Liu, J.; Yang, Z. Numerical study on transonic shock oscillation suppression and buffet load alleviation for a supercritical airfoil using a microtab. Eng. Appl. Comput. Fluid Mech. 2016, 10, 529-544. [CrossRef]

40. Caruana, D.; Mignosi, A.; Correge, M.; Le Pourhiet, A. Buffeting Active Control in Transonic Flow. In Proceedings of the 21st AIAA Applied Aerodynamics Conference, American Institute of Aeronautics and Astronautics, Orlando, FL, USA, 23-26 June 2003. [CrossRef]

41. Caruana, D.; Mignosi, A.; Robitaillié, C.; Corrège, M. Separated Flow and Buffeting Control. Flow Turbul. Combust. 2003, 71, 221-245. [CrossRef]

42. Ferman, M.A.; Huttsell, L.J.; Turner, E.W. Experiments with Tangential Blowing to Reduce Buffet Response on an F-15 Model. J. Aircr. 2004, 41, 903-910. [CrossRef]

43. Abramova, K.A.; Khairullin, B.M.A.; Petrov, K.G.; Potapchik, A.V.; Soudakov, V.G. Buffet Delay on Transonic Airfoil by Tangential Jet Blowing. In Proceedings of the 7th European Conference for Aeronautics and Space Sciences (Eucass), Milan, Italy, 3-6 June 2017.

44. Abramova, K.A.; Ryzhov, A.A.; Sudakov, V.G.; Khairullin, K.G. Numerical modeling of transonic buffeting and its control by means of tangential jet blowing. Fluid Dyn. 2017, 52, 329-335. [CrossRef]

45. Abramova, K.A.; Petrov, A.V.; Potapchick, A.V.; Soudakov, V.G. Investigations of Transonic Buffet Control on Civil Aircraft Wing with the Use of Tangential Jet Blowing. AIP Conf. Proc. 2016, 1770, 020017. [CrossRef]

46. Abramova, K.A.; Soudakov, V.G. Numerical optimization of flow control by tangential jet blowing on transonic airfoil. In Proceedings of the 31st Congress of the International Council of the Aeronautical Sciences, Belo Horizonte, Brazil, 9-14 September 2018. [CrossRef]

47. Grossi, F.; Braza, M.; Hoarau, Y. Prediction of Transonic Buffet by Delayed Detached-Eddy Simulation. AIAA J. 2014, 52, 2300-2312. [CrossRef]

48. Deck, S. Numerical Simulation of Transonic Buffet over a Supercritical Airfoil. AIAA J. 2005, 43, $1556-1566$. [CrossRef]

49. Chen, L.W.; Chang-Yue, X.U.; Xi-Yun, L.U. Numerical investigation of the compressible flow past an aerofoil. J. Fluid Mech. 2010, 643, 97-126. [CrossRef]

50. Soda, A. Numerical Investigation of Unsteady Transonic Shock/Boundary-layer Interaction for Aeronautical Applications. Ph.D. Thesis, Fakultät für Mathematik, Informatik und Naturwissenschaften, Aachen, Germany, 2007.

51. Medida, S.; Baeder, J. Numerical Prediction of Static and Dynamic Stall Phenomena Using the $\gamma$ - $\overline{\operatorname{Re} \theta t}$ Transition Model. American Helicopter Society 67th Annual Forum. Available online: https://www.researchgate.net/publication/288661255_Numerical_prediction_of_static_and_dynamic_ stall_phenomena_using_the_g-Retht_transition_model (accessed on May 2011).

52. Kitamura, K.; Shima, E. Towards shock-stable and accurate hypersonic heating computations: A new pressure flux for AUSM-family schemes. J. Comput. Phys. 2013, 245, 62-83. [CrossRef]

53. Economon, T.D.; Francisco, P.; Sean, R.C.; Trent, W.L.; Juan, J.A. SU2: An Open-Source Suite for Multiphysics Simulation and Design. AIAA J. 2015, 54, 828-846. [CrossRef]

54. McDevitt, J.B.; Okuno, A.F. Static and Dynamic Pressure Measurements on a NACA0012 Airfoil in the Ames High Reynolds Number Facility. NASA TP-2485; June 1985. Available online: https://www.mendeley.com/catalogue/static-dynamic-naca-0012-airfoil-ames-highreynolds-number-facility-naca-0012-reynolds-number-facili/ (accessed on June 1985).

55. Ribeiro, A.F.; Konig, B.; Singh, D.; Fares, E.; Zhang, R.; Chen, H. Buffet Simulations with a Lattice-Boltzmann based Transonic Solver. In Proceedings of the 55th AIAA Aerospace Sciences Meeting, American Institute of Aeronautics and Astronautics, Grapevine, TX, USA, 9-13 January 2017. [CrossRef]

56. Raveht, D.E. Numerical Study of an Oscillating Airfoil in Transonic Buffeting Flows. AIAA J. 2009, 47, 505-515. [CrossRef] 
57. Iovnovich, M.; Raveh, D.E. Reynolds-Averaged Navier-Stokes Study of the Shock-Buffet Instability Mechanism. AIAA J. 2012, 50, 880-890. [CrossRef]

58. Lee, B.H.K. Oscillatory shock motion caused by transonic shock boundary-layer interaction. AIAA J. 1990, 28, 942-944. [CrossRef] 